- Death from odontogenic sepsis is rare but still a reality.

- Odontogenic sepsis is the most common cause of Ludwig's angina.

- Ludwig's angina and other cervicofacial infections present more severely in those with underlying systemic disease.

- Early surgical drainage, aggressive intravenous antimicrobial therapy and supportive care are imperative.

\title{
Death from overwhelming odontogenic sepsis: a case report
}

\author{
L. Carter ${ }^{1}$ and E. Lowis ${ }^{2}$
}

\begin{abstract}
A case of fatal Ludwig's angina from an odontogenic origin complicated by chronic lymphocytic leukaemia is presented. This case highlights that death from odontogenic infection is a reality, particularly in those with systemic disease causing immunocompromise. Early surgical intervention, aggressive intravenous antimicrobial therapy and supportive care is imperative.
\end{abstract}

\section{INTRODUCTION}

Odontogenic infection is the most common cause of Ludwig's angina. ${ }^{1,2}$ Mortality associated with Ludwig's angina has reduced with the routine use of antimicrobials as an adjunct to surgical drainage of head and neck tissue spaces. Up to $35 \%$ of patients presenting with Ludwig's angina have an underlying systemic disease that may have contributed to immunocompromise. ${ }^{1}$

\section{CASE REPORT}

A 67-year-old man presented to Accident and Emergency at Hull Royal Infirmary after emergency transfer from another hospital. He had a three day history of increasing neck swelling and difficulty breathing. His voice was hoarse and

\footnotetext{
${ }^{1 *}$ Specialist Registrar, Oral and Maxillofacial Surgery, Clarendon Way, Leeds, LS2 9LU; ${ }^{2}$ Senior House Officer, Oral and Maxillofacial Surgery, Hull Royal Infirmary, Anlaby Road, Hull, HU3 2JZ

${ }^{*}$ Correspondence to: Dr Lachlan Carter lachlan_carter@hotmail.com
}

\section{Refereed Paper}

Accepted 14 December 2006

DOI: $10.1038 /$ bdj.2007.784

${ }^{\circledR}$ British Dental Journal 2007; 203: 241-242 his sentences limited due to breathlessness. He reported a sore throat, swelling of his face and tongue but no dental pain. He had a past medical history of angina, nasal polyps and chronic lymphocytic leukaemia.

On presentation he was tachycardic (heart rate $126 \mathrm{bpm}$ ), tachypnoeic (respiratory rate 29/minute), pyrexial (temperature $38.9^{\circ} \mathrm{C}$ ), and normotensive with oxygen saturation of $94 \%$ on $\mathrm{FiO}_{2} 0.98$. He was stridulous and had a large, fluctuant, erythematous swelling of the submental and bilateral submandibular spaces extending from the lower border of mandible to the clavicles. The erythema extended over his anterior chest wall (Fig. 1). He exhibited facial and periorbital oedema and erythema. Intra-oral examination revealed multiple carious and periodontally involved teeth. His tongue and floor of mouth were soft and not enlarged. A provisional diagnosis of submental abscess was made. Chlorpheniramine, intravenous hydrocortisone, benzyl penicillin, metronidazole and coamoxiclav were already given before arrival. Nebulised adrenaline was administered and he proceeded to theatre for surgical incision and drainage.
After an awake fibre-optic endotracheal intubation the neck swelling was incised using three access incisions and the submental and bilateral submandibular and sublingual spaces explored. Three drains were placed and serosanguinous fluid was drained (Fig. 2). Dental caries was evident in both lower second molar teeth and the upper right central incisor tooth which were therefore extracted.

He remained intubated and was transferred to ITU. Blood gases revealed a severe metabolic acidosis. Over the following 24 hours he became hypotensive and required inotropic support with

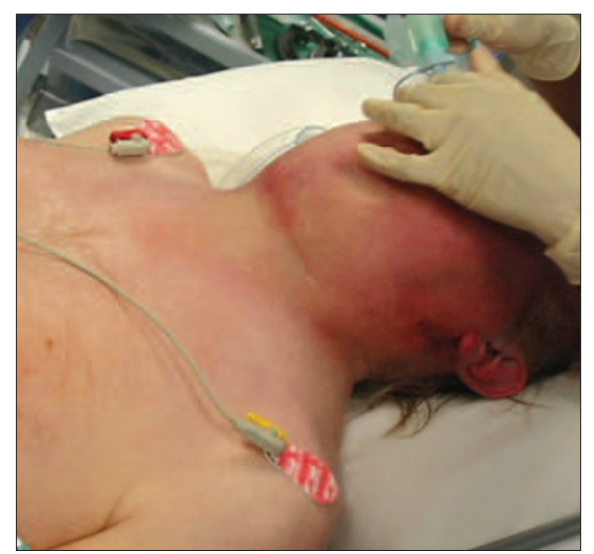

Fig. 1 Neck swelling and chest wall erythema 


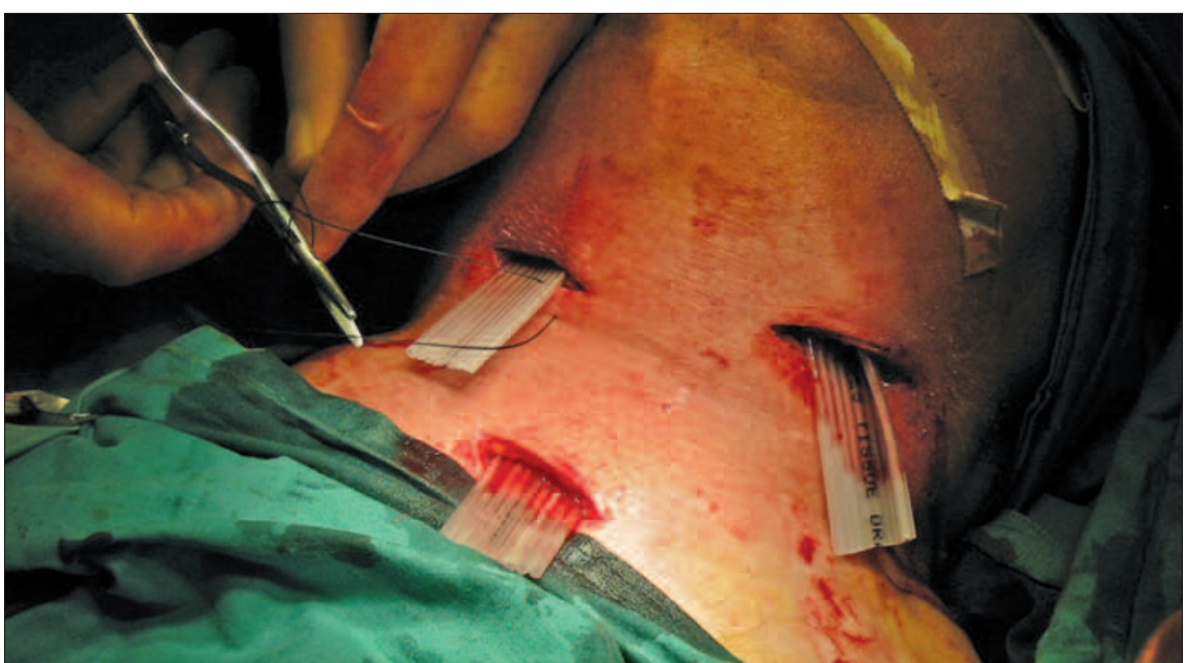

Fig. 2 Access incisions and drains

noradrenaline. The metabolic acidosis worsened despite the ventilatory support, bicarbonate infusions and haemofiltration. As a result of multi-system organ failure he continued to deteriorate and died.

Blood investigations at presentation revealed a white cell count (WCC) of $161.9 \times 10^{9} / \mathrm{L}$ which increased to 570.3 $\mathrm{x} 10^{9} / \mathrm{L}$ over the next 24 hours. A blood film indicated that the high WCC was mostly small round lymphocytes consistent with chronic lymphocytic leukaemia. Some larger lymphocytes were also present which may have been reactive or transformed to large cell lymphoma cells. Group A haemolytic streptococcus was grown from blood cultures and a neck wound swab yielded a scanty growth of streptococcus viridans.

Post mortem examination confirmed a submental/submandibular cellulitis (Ludwig's angina) of streptococcal aetiology as the cause of death and that impaired immunity associated with the underlying leukaemia/lymphoma was a contributary factor.

\section{DISCUSSION} rare. $^{3}$ Progression to Ludwig's angina increases the risk of mortality. With routine use of intravenous antimicrobial therapy as an adjunct to surgical incision and drainage the mortality associated with Ludwig's angina is now less than $10 \% .^{1}$ Odontogenic infection is the most common cause of Ludwig's angina. ${ }^{1,2}$

of those presenting with Ludwig's angina 35\% have an associated underlying systemic disease, most commonly diabetes mellitus. ${ }^{1}$ Myeloproliferative disorders have been reported in 1.2\% of cases. ${ }^{1}$ Chronic lymphocytic leukaemia (CLL) has not been reported as an associated condition before. CLL is a
Death from isolated dental infection is chronic lympho-proliferative disorder with features similar to a low grade lymphoma. In the later stages of CLL, infection can become life-threatening and in this case reduced resistance to infection led to a more severe and ultimately fatal presentation of Ludwig's angina. Those with underlying systemic disease are also more likely to have more severe complications. ${ }^{1,2}$

This case highlights the need for regular dental care in patients with underlying systemic disease in order to prevent more severe presentations of potentially life threatening dental sepsis. Unfortunately the majority of these patients are elderly and are less likely to present for regular dental check ups. ${ }^{4}$

This case is also a stark reminder that dental sepsis can still be fatal, particularly in the presence of systemic disease contributing to immunocompromise. Ludwig's angina and other cervicofacial infections present more severely in patients with underlying systemic disease. Therefore early aggressive surgical drainage, adjunctive intravenous antimicrobial therapy and supportive care are prerequisite.

1. Huang T T, Liu T C, Chen P R et al. Deep neck infection: analysis of 185 cases. Head Neck 2004; 26: 854-860.

2. Bross-Soriano D, Arrieta-Gomez J R, Prado-Calleros $\mathrm{H}$ et al. Management of Ludwig's angina with small neck incisions: 18 years' experience. Otolaryngol Head Neck Surg 2004; 130: 712-717.

3. Currie W J, Ho V. An unexpected death associated with an acute dentoalveolar abscess - report of a case. Br J Oral Maxillofac Surg 1993; 31: 296-298.

4. Haughney M, Devennie J, Macpherson L et al. Integration of primary care dental and medical services: a three year study. Br Dent J 1998; 184: 343-347. 